\title{
Acciones para reducir diferencias de reconciliación entre el mineral recibido y el enviado a molienda
}

\author{
Actions to reduce reconciliation differences between received mineral \\ and sent to mill
}

Johans Herrera Valdez ${ }^{1}$, Jaime César Mayorga Rojas ${ }^{2}$

Recibido: Enero 2020 - Aprobado: Junio 2020 - Publicado: Junio 2020

\begin{abstract}
RESUMEN
En toda operación minera es necesario un sistema de reconciliación, el cual permita medir el desempeño de los procesos involucrados en la obtención del producto final, desde los modelos de reservas-recursos, pasando por el diseño de mina, planeamiento de minado, producción minera, hasta culminar en el proceso metalúrgico.

Es mediante la relación entre el mineral recibido (mineral molido) con el mineral enviado a molienda (modelo corto plazo), que resultarán factores de reconciliación para tonelaje, ley y metal. Posterioremente, serán evaluados en un periodo de tiempo mensual, trimestral y/o anual, y servirán de ayuda para afinar los procesos y subprocesos del modelo de corto plazo, asi como el proceso de minado y el proceso metalúrgico. Los resultados se verán reflejados en la reducción de las diferencias de los factores de reconciliación para el tonelaje, ley y metal, esta reducción debe ser soportada en el tiempo; es decir, un sistema de reconciliación minera debe ser parte de la mejora continua en toda la cadena de valor del proceso minero.
\end{abstract}

Palabras claves: Reconciliación minera; contenido metal; polígonos de mineral; stockpiles; modelo de corto plazo (grade control).

\begin{abstract}
In all mining operations, a reconciliation system is necessary, which allows measuring the performance of the processes involved in obtaining the final product, from the reserve-resource models, through mine design, mine planning, mining production, until culminating in the metallurgical process.

It is through the relationship between the ore received (ground ore) with the ore sent to grinding (short-term model), that reconciliation factors will result for tonnage, grade and metal. Subsequently, they will be evaluated in a monthly, quarterly and / or annual period of time, and will help to refine the processes and sub-processes of the short-term model, as well as the mining process and the metallurgical process. The results will be reflected in the reduction of the differences in the reconciliation factors for tonnage, grade and metal, this reduction must be supported over time; In other words, a mining reconciliation system must be part of continuous improvement throughout the value chain of the mining process.
\end{abstract}

Keywords: Mining reconciliation; metal content; mineral polygons; stockpiles; short term model (grade control).

\footnotetext{
${ }^{1}$ Sindicato Minero de Orcopampa S.A. Calle Lizardo Alzamora Oeste 220 - Lima 27. E-mail: johans.herrera@unmsm.edu.pe ${ }^{2}$ Docente de la EPIG, Facultad de Ingeniería Geológica, Minera, Metalúrgica y Geográfica, Universidad Nacional Mayor de San Marcos. Lima, Perú. Av. Venezuela S/N Cuadra 34 Ciudad Universitaria - Lima 01. E-mail: jaime.mayorga@unmsm.edu.pe 


\section{INTRODUCCIÓN}

El proceso de identificar, analizar y gerenciar las variaciones entre lo planeado y los resultados actuales, se conoce como reconciliación minera, son las oportunidades de mejora en el desempeño de una operación minera.

Este proceso, involucra la recolección de data de tonelajes, leyes y contenido metal; data idealmente de fuentes independientes, ejemplos son: data de exploraciones, data de muestreo de perforación de blastholes (pozos de producción), data del proceso minero, data del proceso metalúrgico, entre otros. Esta data puede ser comparada por factores y límites o rangos permisibles.

La medición y el análisis de la data de una manera detallada permitirán en una operación minera diseñar e implementar procesos de mejora en toda la cadena de valor del proceso minero, como en la estimación de reservas-recursos, el diseño de mina, el planeamiento de minado, el control de leyes, el movimiento del mineral y el procesamiento para el producto final.

\section{METODOS}

\subsection{Metodología: reconciliación entre el mineral recibido y el enviado a planta}

Reconciliación, es el proceso clave que permite determinar la capacidad de una operación minera para producir tonelaje, ley y contenido metal que fueron estimados en el modelo de reservas-recursos.

Los procesos y subprocesos que intervienen en una buena o mala reconciliación y las relaciones entre ellas se muestran en la Figura 1.
Para el presente artículo se evaluará la relación entre el mineral recibido (producido en planta, molido) y el mineral enviado a planta (modelo de corto plazo, grade control).

La información del mineral recibido en molienda usualmente se extrae de los balances metalúrgicos, que son los reportes oficiales de la producción de la operación minera. El mineral enviado a molienda es el agotamiento del modelo de corto plazo (grade control), mineral de polígonos a molienda más mineral de stockpiles a molienda. Figura 2, proceso de minado.

Las relaciones de tonelaje $(\mathrm{t})$, ley $(\mathrm{g})$ y contenido metal (m) entre el mineral recibido en molienda y el mineral enviado a molienda, son usados para desarrollar factores de reconciliación: F2t, F2g, F2m (Parker, H., 2013).

F2 = Recibido en Molienda / Enviado a Molienda

o

$$
\text { F2 = Planta / Modelo de Corto Plazo }
$$

\section{RESULTADOS}

Para analizar los resultados, debemos hacer uso de gráficas para visualizar las diferencias de los factores de reconciliación en un determinado periodo de tiempo. En el siguiente gráfico con datos supuestos, para el factor de reconciliación F2 metal observamos que a partir del último semestre del año 2017 se tuvo una tendencia de reducción entre el material recibido (planta) y el enviado (modelo de corto plazo) (Figura 3).

Los problemas y causas frecuentes de las diferencias de reconciliación entre el mineral recibido y el enviado a planta, los podemos dividir dentro de tres procesos: el

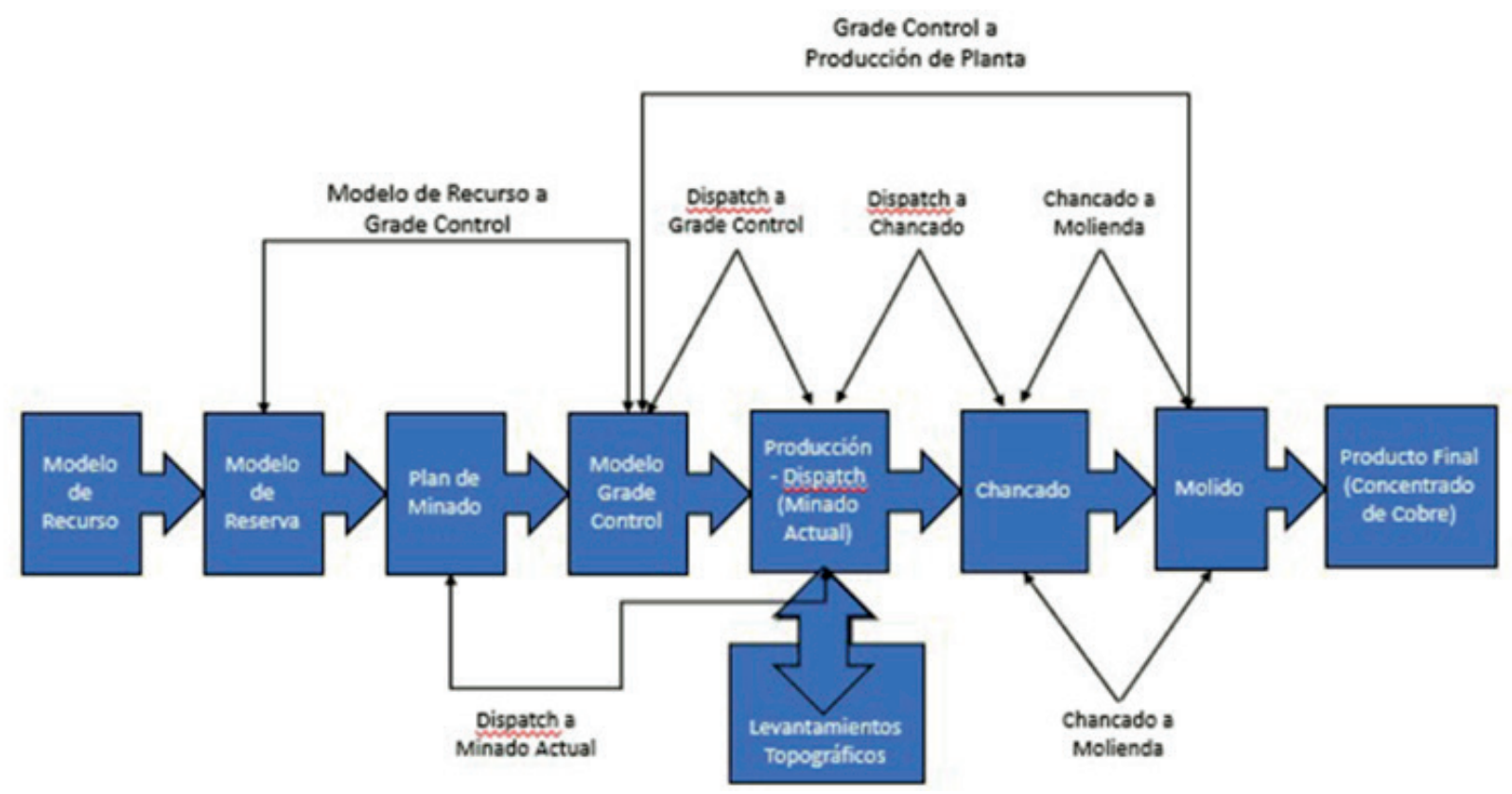

Figura 1. Reconciliación a través de la cadena de valor de la mina y un rango de comparaciones Fuente: (Morley \& Hargreaves, 2014). 


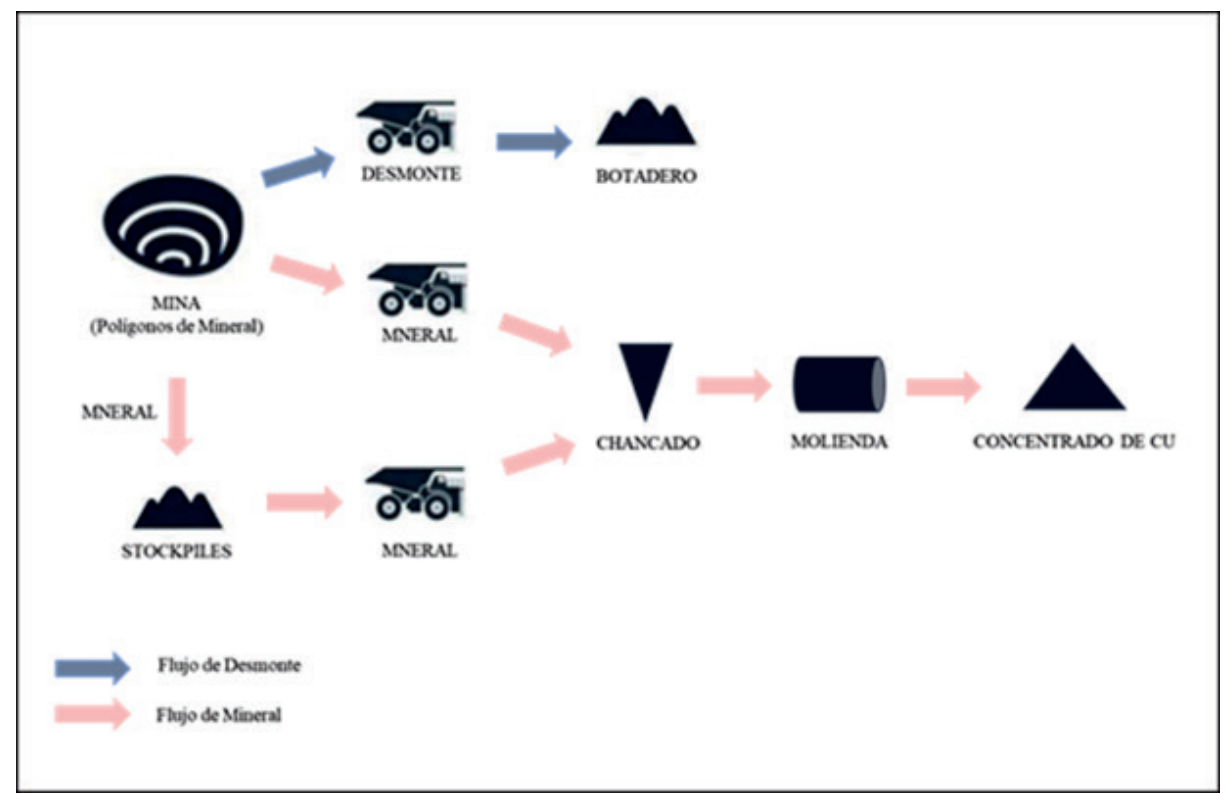

Figura 2. Proceso de minado. Fuente: Elaboración propia

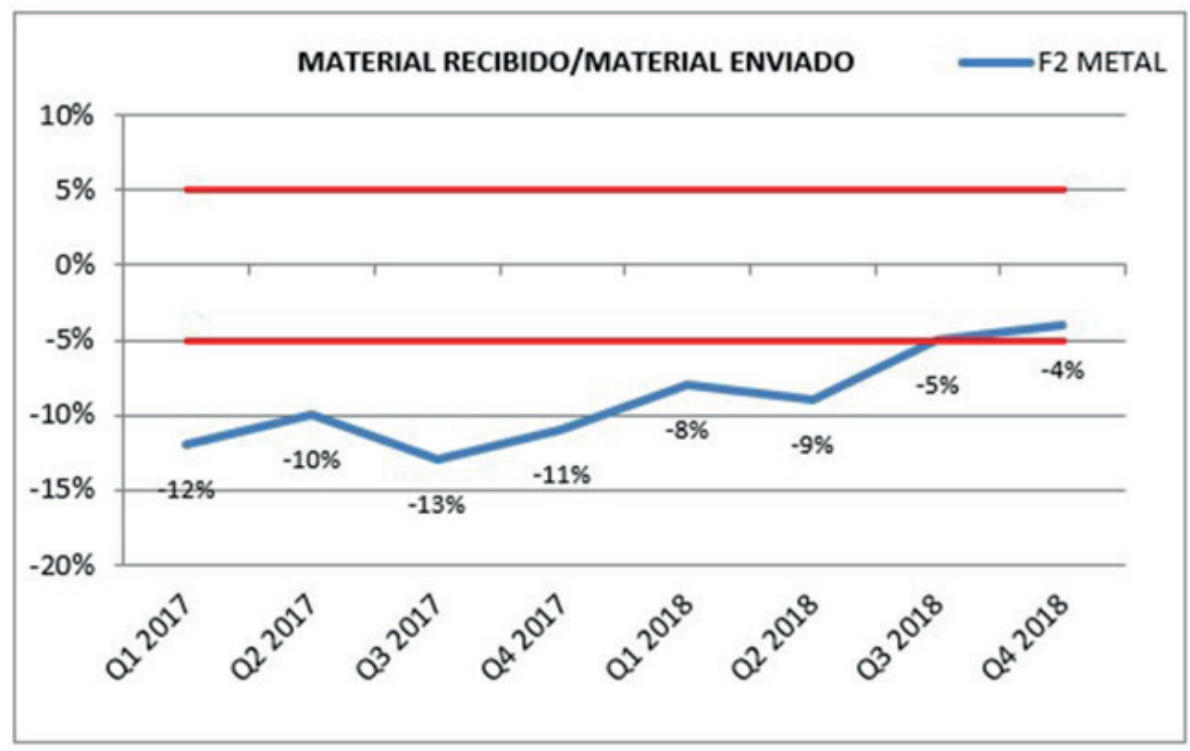

Figura 3. Gráfico trimestral, se observa que a partir del último semestre del año 2017 se tuvo reducción de reconciliación entre el material recibido y el material enviado para F2 metal. Fuente: Elaboración Propia

modelo de corto plazo (grade control), el minado y el procesamiento en planta. A continuación, abordaremos principalmente los problemas y causas para el modelo de corto plazo (grade control) y para el minado según (Morley \& Hargreaves, 2014).

\subsection{Modelo de corto plazo (grade control)}

3.1.1 Bajo conocimiento de la geología del depósito mineral.

El conocimiento geológico es importantísimo pues ayudará en la interpretación de las diferentes litologías, dominios de estimación.

Un pobre entendimiento de los dominios litológicos $\mathrm{y}$ sus contactos, controles estructurales, alteración y mineralización; puede tener un profundo efecto que será reflejado en la estimación y posteriormente en la reconciliación.

Donde la reconciliación muestra variación espacial en la ubicación de la mineralización o casos de dilución y/ pérdida de mineral, es probable que exista desviaciones con la interpretación geológica de las zonas mineralizadas.

\subsubsection{Efecto pepita}

Un efecto pepita alto significa que las muestras tomadas tienen alta variabilidad debido a la heterogeneidad del depósito mineral.

El efecto pepita, además de la heterogeneidad del depósito mineral, puede deberse a otros factores como: el 
soporte usado en el muestreo, errores en el procedimiento de muestreo, error en la ubicación de los muestreos, entre otros (Emery, X., s. f.).

Intentar minar con alta selectividad una zona de alto efecto pepita, traerá como consecuencia pobres reconciliaciones como la estimación de leyes localmente inexactas.

Esto puede conllevar a errores en la clasificación del mineral y variabilidad en la molienda.

Para reconciliación, en un ambiente de efecto pepita alto, los resultados deben ser tratados en largo periodos de tiempo o grandes volúmenes.

\subsubsection{Errores de muestreo}

Debido a errores en la perforación, muestreo, preparación y análisis puede resultar en pobre precisión y exactitud de los resultados de leyes. Por lo tanto, una pobre precisión mostrará un efecto pepita alto en el análisis de los variogramas de estimación.

Un tema particular en el control de leyes es el uso de blastholes para muestreo. El espaciamiento, tamaño, ubicación y orientación de blastholes son todos diseñados para optimizar la voladura y no para optimizar el control de leyes.

Blastholes pueden resultar en precisiones pobres en los análisis de leyes, principalmente en zonas de alto efecto pepita.

\subsubsection{Pobres ángulos de perforación}

Donde el control de leyes use blastholes para el muestreo, la dirección de perforación en zonas de mineral no puede ser optimizada. Lo ideal que la dirección de perforación sea perpendicular a la dirección e inclinación de las zonas de mineralizadas. Blastholes verticales no son adecuados en mineralización subvertical.

\subsubsection{Incorrectas leyes de corte de mineral}

El uso de cut-off de ley inapropiados para definir límites de mineral (para valores altos y bajos), puede resultar en diferencias de reconciliación en zonas donde el mineral no es altamente visual.

- Malas prácticas de estimación

El uso de técnicas inapropiadas de estimación puede incurrir en un modelo impreciso, y por lo tanto en una pobre reconciliación.

- Incorrectos mediciones de densidad

Las mediciones de densidad para cálculo de tonelajes deben ser tomados en todas las rocas (dominios) teniendo en cuenta zonas de mineralización (zonas de intemperismo, óxidos, transición, sulfuros para depósitos de $\mathrm{Cu}$ ) y estilos de mineralización, para asegurar la exactitud del modelo de reserva inicial.

Pobres mediciones resultarán en una reconciliación subestimada en tonelaje.
- Diseño de polígonos de mineral

El mejor modelo de corto plazo puede ser inservible, si los polígonos de mineral son diseñados y marcados inapropiadamente, errores de topografía, errores humanos, desplazamiento del mineral por voladura, pueden conllevar a una pobre reconciliación. El control visual durante el minado es la mejor forma de prevenir estos problemas.

- $\quad$ Errores en la base de datos

Problemas en la base de datos reflejará pobre reconciliación e incertidumbre en el control de leyes. Los errores humanos son comunes.

\subsection{Minado}

\subsubsection{Voladura}

La voladura de acuerdo a mayor o menor factor de potencia desplazará el mineral ocasionando dilución, perdida de mineral y/o errores en la clasificación de mineral.

El desplazamiento debe ser monitoreado corrigiendo las marcas de polígonos en campo y en el sistema dispatch.

\subsubsection{Dirección de minado}

La dirección de minado paralelo a la mineralización hace más difícil el control visual del límite del mineral con el desmonte, lo ideal que el minado sea transversal a la mineralización para mejor control de los límites de mineral.

\subsubsection{Dilución de mineral}

Principalmente por polígonos de mineral demasiado pequeños en contacto con polígonos de desmonte, originando una mezcla al momento de minar.

El desplazamiento de polígonos post voladura origina pérdida de mineral (porción de polígonos de mineral sobre polígonos de desmonte), dilución de mineral (porción de polígonos de desmonte sobre polígonos de mineral), y errores de clasificación en el tipo de mineral.

\subsubsection{Errores topográficos}

Los errores topográficos pueden causar pobres reconciliaciones locales, estos errores pueden envolver en malas ubicaciones de perforaciones y por tanto malas ubicaciones de leyes, errores en diseños de límites de mineral para minado, errores en la generación de superficies y sólidos de minado para volúmenes de reconciliación, entre otros.

\subsubsection{Destino de camiones}

Los errores humanos al momento de asignaciones erróneas de polígonos origen y destinos de mineral, por ejemplo, errores de operador de pala al enviar camiones con material estéril a stockpiles o a chancadora.

\subsection{Planta}

Con respecto al procesamiento de planta, tenemos problemas relacionados a: balance de material basado en muestreos no probabilísticos, exactitud analítica, procesos 
de ciclos desconocidos o mal llevados, calibraciones de pesómetros y flujómetros, pobre muestreo de laboratorio metalúrgico; donde se recomienda la evaluación de los protocolos de trabajo incluyendo las calibraciones periódicas de los equipos de medición.

\section{DISCUSION}

\subsection{Discusión: acciones para reducir diferencias}

Las acciones para reducir las diferencias de reconciliación entre el material recibido y el enviado a molienda (planta) son resumidos en la Tabla 1, estas acciones son principalmente para el proceso de modelo de corto plazo (grade control) y para el minado. En el procesamiento de planta las acciones recomendadas son la revisión de todas las entradas para los cálculos del balance metalúrgico, evaluación de protocolos y calibraciones de equipos de medición periódicas son indispensables.

Para evaluar si las acciones tomadas han sido efectivas debemos realizar gráficas de reconciliación en un mediano periodo de tiempo: trimestral, semestral, anual; siendo el objetivo reducir las diferencias y mantenerlas dentro de un rango aceptable ( $\pm 5 \%$ ) (Figura 4 , Figura 5 y Figura 6$)$.

\section{CONCLUSIONES}

- En toda operación minera es necesario implementar reconciliación que permita analizar y explicar las diferencias entre las comparaciones de lo programado y producido, para saber qué medidas de reducción tomar a futuro, identificando $y$ mejorando los procesos y subprocesos del proceso minero.

- Estas diferencias indican pérdidas de mineral en el proceso, debiendo revisar, evaluar y mejorar procesos desde el muestreo, estimación de modelos de reservas - recursos, modelos de corto plazo (grade control), operación minera (voladura, carguío de mineral, asignaciones de orígenes, destinos, balanzas), y proceso metalúrgico.

- En las acciones para reducir las diferencias, éstas son principalmente para el modelo de corto plazo (grade control) y para el minado; con respecto al procesamiento de planta, las acciones recomendadas son la revisión de las entradas en el balance metalúrgico.

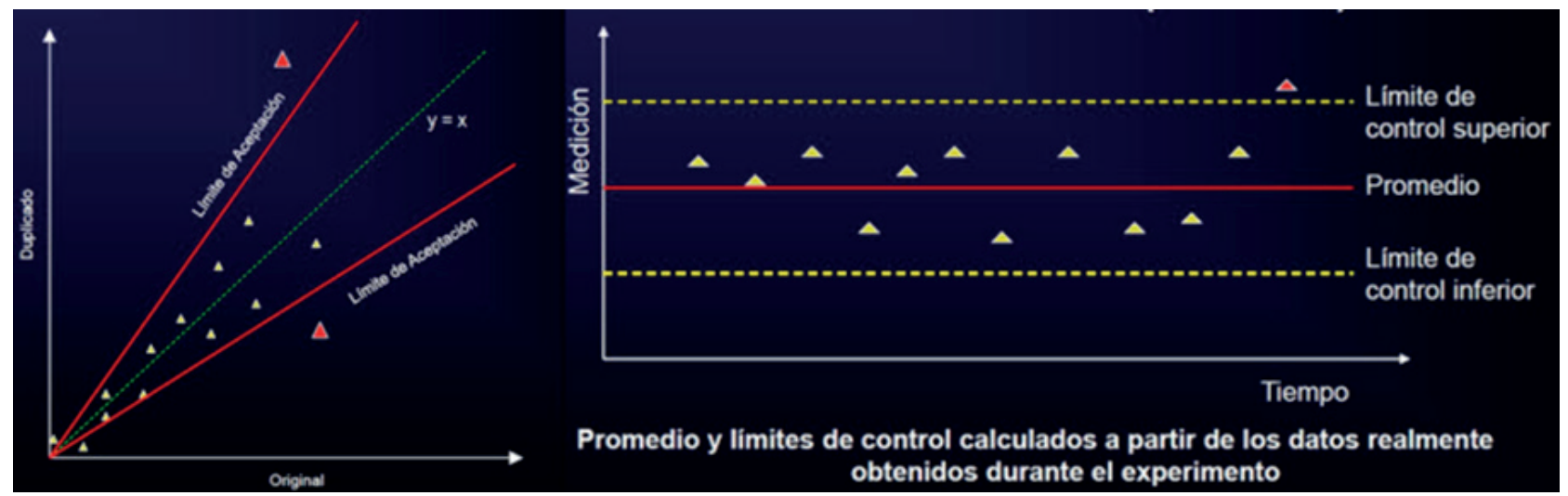

Figura 4. Implementación de programas de control de calidad de leyes. Gráficos de QAQC para el control de leyes. Izquierda, Gráfico de máximos - mínimos para medir la precisión (Scott Long). Derecha, Gráfico con límites de control a partir de la desviación estándar para medir la exactitud (Shewhart). Fuente: (Simon, A., 2016).

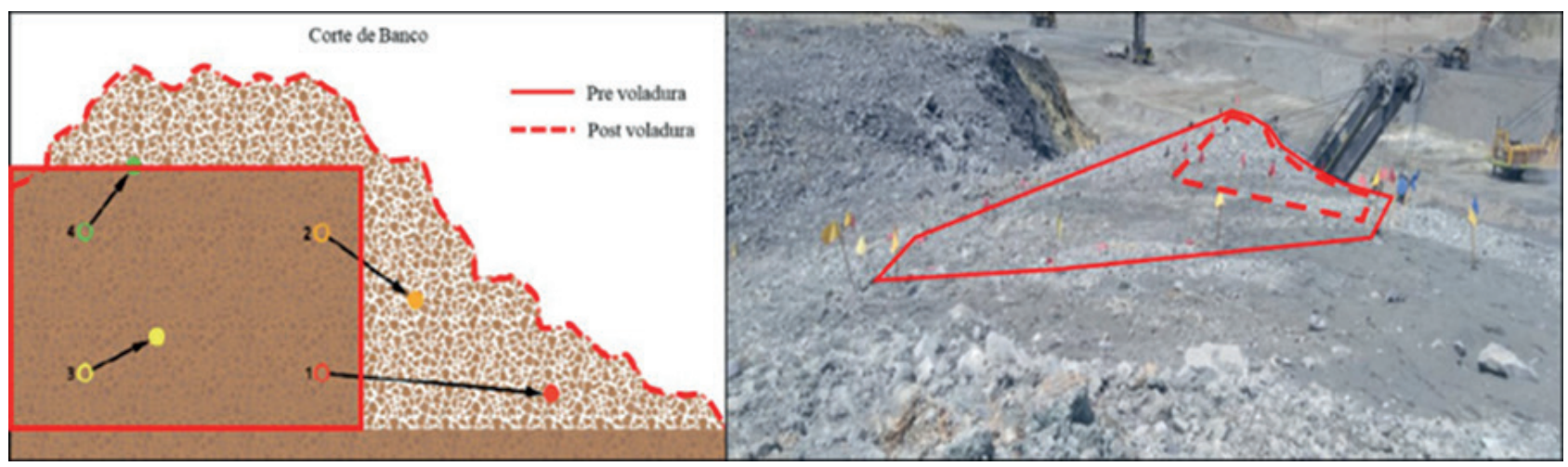

Figura 5. Metodología Blast Movement Technology, para desplazamiento de polígonos post voladura. Fuente: Izquierda: (3D Blast Movement Monitoring | BMT, s. f.). Derecha: Elaboración Propia 


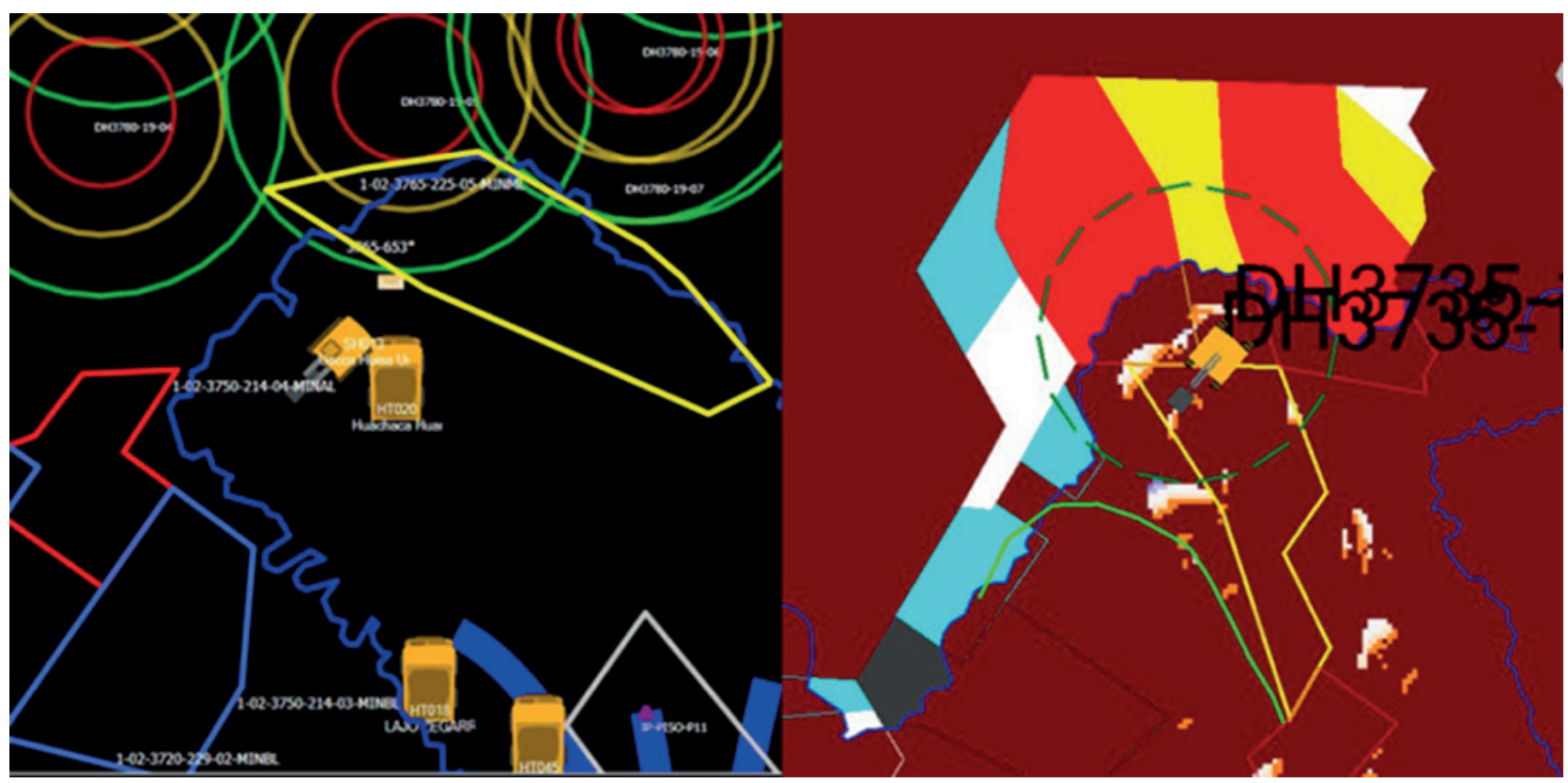

Figura 6. Sistema de monitoreo GPS de carguío y minado (dispatch-provision).

Fuente: Elaboración propia

Tabla 1. Problemas/Causas y Acciones de mejora para reducir diferencias de Reconciliación entre el Mineral Recibido y el Enviado a Planta

\begin{tabular}{ll}
\hline Problemas / Causas Acciones para reducir diferencias entre el Mineral Recibido y el Enviado a Planta \\
\hline Modelo de Corto Plazo (Grade Control)
\end{tabular}

Bajo conocimiento de la geología del Plan de perforación infill en zonas de blancos de información. depósito mineral.

Interacción de Geólogos de Grade Control con Geólogos de Exploración, afinación de criterios.

Mapeo de Taludes para ajustar la interpretación en combinación con el logueo de detritos de los Blastholes.

Buen logueo de Blastholes en zonas de contactos, considerar 2 tramos.

Efecto Pepita

Revisar y definir el soporte usado para las mediciones. Soportes más voluminosos conducen a una reducción del efecto pepítico.

Revisar y definir espaciamientos de muestreos.

Eliminar errores de medición de datos.

Eliminar errores de ubicación de datos.

Errores de Muestreo

Revisión de método y protocolos de muestreos.

Programas de QAQC para controlar y corregir imprecisiones e inexactitudes en el muestreo. Fig. $\mathrm{N}^{\circ} 4$.

Pobres Ángulos de Perforación

Incorrectas Leyes de Corte de Mineral

Programa de perforación dedicado al control de leyes, buenos resultados, pero aumentará tiempos y costos.

Correctos Análisis Exploratorios de Data (EDA's) de leyes de muestreo.

Definición de leyes de corte por dominio de estimación.

Malas Prácticas de Estimación

Incorrectas Mediciones de Densidad

Revisión y definición de parámetros de estimación por personal calificado (QP's).

Revisión de método y protocolos de muestreos.

Uso de valores de densidad calculadas (estimadas) y no asignadas.

Homogenizar las metodologías de cálculo de densidad en todos los Modelos: Reservas, Corto Plazo y Grade Control.

Corte de valores altos de acuerdo a un EDA validado.

Diseño de Polígonos de Mineral

Forma y tamaños adecuados al equipo de carguío y dirección de minado.

Evitar ángulos agudos y polígonos pequeños.

Supervisar el marcado de polígonos en campo.

Implementar metodologías para desplazamiento de polígonos post voladura. Fig. N5․

Minado

Voladura

Diseños de Voladura evitando desplazamiento horizontal.

Estudios de factor de potencia para obtención de finos necesarios para la molienda. 
Tabla 1: Continuación

\begin{tabular}{|c|c|}
\hline Problemas / Causas & Acciones para reducir diferencias entre el Mineral Recibido y el Enviado a Planta \\
\hline Dirección de Minado & Evitar en lo posible minado paralelo a contactos de mineral - desmonte, se recomienda minados transversales. \\
\hline \multirow[t]{3}{*}{ Dilución de Mineral } & $\begin{array}{l}\text { Diseño de polígonos (forma y tamaño) adecuados al equipo de carguío, dirección de minado, contactos de mineral - } \\
\text { desmonte. }\end{array}$ \\
\hline & Supervisión de marcado de polígonos en campo y remarcado según control visual. \\
\hline & Implementación de un sistema de monitoreo de desplazamiento de voladura para marcado de polígonos desplazados. \\
\hline \multirow[t]{3}{*}{ Errores Topográficos } & Revisión de protocolos de levantamientos topográficos. \\
\hline & Supervisión periódica de levantamientos topográficos. \\
\hline & Validación y aprobación por Ingenieros Senior de Planeamiento de las superficies y sólidos de minado. \\
\hline \multirow[t]{3}{*}{ Destino de Camiones } & Entrenamiento a operadores de equipos de carguío (palas, cargadores) en los diferentes tipos de polígonos de mineral. \\
\hline & Automatización del proceso de carguío, minado. \\
\hline & Implementación de un sistema de monitoreo GPS de carguío y minado (dispatch-provision). Fig. № 6 . \\
\hline \multicolumn{2}{|l|}{ Planta } \\
\hline $\begin{array}{l}\text { Balance de material basado en muestreos } \\
\text { no probabilisticos }\end{array}$ & Evaluación de los protocolos de trabajo incluyendo las calibraciones periódicas de los equipos de medición \\
\hline \multicolumn{2}{|l|}{ Exactitud Analítica } \\
\hline \multicolumn{2}{|l|}{$\begin{array}{l}\text { Procesos de ciclos desconocidos o mal } \\
\text { llevados }\end{array}$} \\
\hline $\begin{array}{l}\text { Calibraciones de Pesómetros y } \\
\text { Flujómetros }\end{array}$ & \\
\hline Pobre muestreo de laboratorio metalúrgico & \\
\hline
\end{tabular}

Fuente: Elaboración Propia

- Las principales acciones para reducir diferencias con respecto al modelo de corto plazo (grade control) son: aumentar y mejorar el plan de perforación infill en zonas de blancos de información, revisar y definir los soportes y espaciamiento en el muestreo, implementar programas de QAQC para controlar y corregir imprecisiones e inexactitudes en el muestreo, correcta definición de leyes de corte por dominio de estimación (Figura 4), uso de valores de densidad calculadas (estimadas) y no asignadas, forma y tamaños de polígonos adecuados al equipo de carguío y dirección de minado, implementación de metodologías para desplazamiento de polígonos post voladura (Figura 5).

- Las principales acciones para reducir diferencias con respecto al minado son: diseños de voladura evitando desplazamiento horizontal, implementación de un sistema de monitoreo de desplazamiento de voladura para marcado de polígonos desplazados, automatización del proceso de carguío y minado, implementando un sistema de monitoreo GPS (Figura 6).

\section{AGRADECIMIENTOS}

Los autores del presente artículo quieren extender sus agradecimientos a la Unidad de Posgrado de la Universidad Nacional Mayor de San Marcos por su apoyo académico.

\section{REFERENCIAS}

$3 D$ Blast Movement Monitoring | BMT. (s. f.). What is blast movement? Recuperado 19 de junio de 2020, de https:// blastmovement.com/blast-monitoring/

Emery, $X$. (s. f.). Geoestadística. Ingeniería de Minas. Facultad de Ciencias Físicas y Matemáticas. Universidad de Chile. Recuperado 19 de junio de 2020, de https://www.u-cursos. cl/usuario/f697544fb48474f4153ef2aafbe4c963/mi_blog/r/ Geoestadistica.pdf

Morley, C., \& Hargreaves, R. (2014, enero 1). Mining Reconciliation - an overview of data collection points and data analysis. Mineral Resource and Ore Reserve Estimation - The AusIMM Guide to Good Practice - Second Edition. https://www.researchgate.net/publication/263770059 Mining_Reconciliation_-_an_overview_of_data_ collection_points_and_data_analysis

Parker, H. (2013). Reconciliation principles for the mining industry: Mining Technology: Vol 121, No 3. https://www.tandfonline. com/doi/full/10.1179/1743286312Y.0000000007

Simon, A. (2016). Aseguramiento de la Calidad: Taller de Aseguramiento y Control de la Calidad en la Exploración Geológica. https://es.scribd.com/document/217351100/04Taller-de-ACC-Control-de-Calidad-pdf 
